\title{
Apatinib-loaded nanoparticles suppress vascular endothelial growth factor-induced angiogenesis and experimental corneal neovascularization
}

This article was published in the following Dove Press journal:

International Journal of Nanomedicine

5 July 2017

Number of times this article has been viewed

\author{
Jung Eun Lee ${ }^{1, *}$ \\ Koung Li Kim ${ }^{2, *}$ \\ Danbi Kim² \\ Yeongju Yeo $^{2}$ \\ Hyounkoo Han ${ }^{3}$ \\ Myung Goo Kim ${ }^{1,4}$ \\ Sun Hwa Kim ${ }^{4}$ \\ Hyuncheol Kim ${ }^{3,5}$ \\ Ji Hoon Jeong ${ }^{1,4}$ \\ Wonhee Suh ${ }^{2}$
}

'School of Pharmacy, Sungkyunkwan University, Suwon, ${ }^{2}$ College of Pharmacy, Chung-Ang University, Seoul, ${ }^{3}$ Department of Chemical and Biomolecular Engineering, Sogang University, ${ }^{4}$ Center for Theragnosis Biomedical Research Institute, Korea Institute of Science and Technology (KIST), ${ }^{5}$ Department of Biomedical Engineering, Sogang University, Seoul, Korea

*These authors contributed equally to this work

Correspondence: ji Hoon Jeong School of Pharmacy, Sungkyunkwan University, 2066 Seobu-Ro, Jangan-Gu,

Suwon 16419, Gyeonggi-Do, Korea

$\mathrm{Tel}+823$ 3। 2907783

Fax +82 3I 2928800

Email jhjeong@skku.edu

Wonhee Suh

College of Pharmacy, Chung-Ang

University, 84 Heukseok-Ro, Dongjak-gu,

Seoul 06974, Korea

Tel +82 28205960

Fax +8228167338

Email wsuh@cau.ac.kr

\begin{abstract}
Pathological angiogenesis is one of the major symptoms of severe ocular diseases, including corneal neovascularization. The blockade of vascular endothelial growth factor (VEGF) action has been recognized as an efficient strategy for treating corneal neovascularization. In this study, we aimed to investigate whether nanoparticle-based delivery of apatinib, a novel and selective inhibitor of VEGF receptor 2, inhibits VEGF-mediated angiogenesis and suppresses experimental corneal neovascularization. Water-insoluble apatinib was encapsulated in nanoparticles composed of human serum albumin (HSA)-conjugated polyethylene glycol (PEG). In vitro angiogenesis assays showed that apatinib-loaded HSA-PEG (Apa-HSA-PEG) nanoparticles potently inhibited VEGF-induced tube formation, scratch wounding migration, and proliferation of human endothelial cells. In a rat model of alkali burn injury-induced corneal neovascularization, a subconjunctival injection of Apa-HSA-PEG nanoparticles induced a significant decrease in neovascularization compared to that observed with an injection of free apatinib solution or phosphate-buffered saline. An in vivo distribution study using HSAPEG nanoparticles loaded with fluorescent hydrophobic model drugs revealed the presence of a substantial number of nanoparticles in the corneal stroma within $24 \mathrm{~h}$ after injection. These in vitro and in vivo results demonstrate that apatinib-loaded nanoparticles may be promising for the prevention and treatment of corneal neovascularization-related ocular disorders.
\end{abstract}

Keywords: apatinib, corneal neovascularization, nanoparticle, vascular endothelial growth factor

\section{Introduction}

The eye is divided into two principal anatomical segments: the anterior and posterior segments. While the posterior segment including the retina and choroid is highly vascularized, the anterior segment, particularly the cornea, is avascular, which allows light to reach the retina. The avascularity of the cornea is maintained by the strict balance between angiogenic and antiangiogenic factors. ${ }^{1}$ However, infection, injury, and inflammation tilt the balance toward corneal neovascularization, which disturbs proper optical performance and can compromise visual acuity. ${ }^{2}$ Current treatments for corneal neovascularization include anti-inflammatory medications, photodynamic therapy, laser photocoagulation, and transplantation of the conjunctiva, limbus, or amniotic membrane. ${ }^{2}$ Unfortunately, all of these medical treatments and surgical interventions have limited clinical efficacy and unwanted side effects.

Vascular endothelial growth factor (VEGF), previously known as vascular permeability factor, plays a prominent role in corneal neovascularization. In the normal cornea, VEGF proteins, which are constitutively expressed in the corneal 
epithelium, endothelium, and vascular endothelial cells of limbal vessels, are sequestered by endogenously expressed soluble VEGF receptors (VEGFRs) to maintain avascularity and transparency. ${ }^{1,3,4}$ Under pathological conditions, VEGF expression substantially increases in the cornea and the VEGF proteins released stimulate angiogenesis in the limbal vascular plexus and invasion of neovessels into the cornea. ${ }^{5-7}$ The importance of VEGF in corneal neovascularization was demonstrated in several recent studies where VEGFneutralizing antibodies substantially regressed blood vessels in the cornea of patients with corneal grafts, viral infection, pterygia, or Stevens-Johnson syndrome..$^{8-14}$

Among the numerous VEGFR subtypes, VEGFR2 plays a major role in mediating VEGF-induced endothelial cell proliferation, migration, capillary tube formation, and permeabilization. ${ }^{15}$ Because VEGF-induced phosphorylation of VEGFR2 activates multiple downstream signaling pathways involved in angiogenesis, receptor tyrosine kinase inhibitors targeting VEGFR2, which were initially developed for treating various cancers, have been suggested as potential therapies for corneal neovascularization. ${ }^{16,17}$ Therefore, we propose that apatinib, a novel and selective inhibitor of VEGFR2 tyrosine kinase, may have beneficial actions against corneal neovascularization. In our previous study, we encapsulated water-insoluble apatinib in nanoparticles composed of human serum albumin (HSA)-conjugated polyethylene glycol (PEG) and demonstrated that the apatinib-loaded HSA-PEG (Apa-HSA-PEG) nanoparticles efficiently reduced VEGF-induced retinal vascular hyperpermeability by blocking VEGF/VEGFR2 signaling. ${ }^{18}$ In this present study, we aimed to characterize the antiangiogenic effect of Apa-HSA-PEG nanoparticles in human endothelial cells and evaluate their preventive effect on alkali burn injury-induced corneal neovascularization in rats.

\section{Materials and methods Synthesis of HSA-PEG conjugate}

The HSA-PEG conjugate was prepared as described in a previous study. ${ }^{18}$ In brief, HSA-PEG conjugates were synthesized by mixing N-hydroxysuccinimide-derivatized methoxy PEG (NHS-mPEG, molecular weight [MW] 5,000, SunBio Inc., Anyang, Korea) and HSA (Sigma, St Louis, MO, USA) at a stoichiometric feed ratio of 20:1 (NHS-mPEG:HSA) in phosphate-buffered saline (PBS). The reaction mixture was incubated at room temperature for $12 \mathrm{~h}$, dialyzed to remove unreacted PEG, and then freeze dried. The MW of HSA-PEG conjugate was determined by gel permeation chromatography using a Waters 626 pump equipped with a
Waters 486 ultraviolet detector and PL aquagel $\mathrm{OH}$ mixed column (Agilent 1100s, Agilent, Santa Clara, CA, USA). The analysis was carried out with PBS as the mobile phase at a flow rate of $1.0 \mathrm{~mL} / \mathrm{min}$.

\section{Preparation and characterization of Apa- HSA-PEG nanoparticles}

To prepare Apa-HSA-PEG nanoparticles, HSA-PEG conjugates and apatinib (LSK Biopharma, Salt Lake City, UT, USA) were dissolved in 50\% aqueous tetrahydrofuran. The solvent was then evaporated under reduced pressure at $100^{\circ} \mathrm{C}$, which formed a thin and transparent film containing HSAPEG conjugates and apatinib. The film was rehydrated in deionized water and sonicated to disperse into self-assembled nanoparticles. The resulting solution was filtered through a $0.8 \mu \mathrm{m}$ cellulose acetate filter unit, lyophilized, and stored at $-20^{\circ} \mathrm{C}$ until needed. For the in vitro and in vivo experiments, Apa-HSA-PEG nanoparticles were reconstructed by dissolving the lyophilized product in PBS. The drug loading content and loading efficiency of the nanoparticles were determined using a Water 626 high-performance liquid chromatography (HPLC) pump equipped with a LiChrospher ${ }^{\circledR}$ 100 RP-18 column (Millipore, Billerica, MA, USA) in an acetonitrile:water $(45: 55, \mathrm{v} / \mathrm{v})$ mobile phase. The flow rate was $0.5 \mathrm{~mL} / \mathrm{min}$. The size and morphology of Apa-HSA-PEG nanoparticles were examined using dynamic light scattering (Zeta Plus, Brookhaven Instrument Co., Holtsville, NY, USA) and transmission electron microscopy, respectively.

\section{In vitro drug release study}

The release of apatinib from Apa-HSA-PEG was monitored using a dialysis method. The freeze-dried Apa-HSA-PEG (10 mg) suspended in $1 \mathrm{~mL}$ of deionized water was placed in the dialysis membrane (MW cutoff 10,000, Spectrum, Rancho Dominguez, CA, USA). The drug release was performed in $20 \mathrm{~mL}$ PBS solution on an orbital shaker at $37^{\circ} \mathrm{C}$. The samples were collected at predetermined time intervals and supplemented with an equal volume of fresh PBS. The concentration of the drug was determined by HPLC analysis.

\section{In vitro angiogenesis assays}

Human umbilical vein endothelial cells (HUVECs) were obtained from Lonza (Walkersville, MD, USA) and cultured in endothelial growth medium-2 (Lonza) at $37^{\circ} \mathrm{C}$ in a humidified incubator from passages three to six. To analyze the inhibitory effects of apatinib on angiogenesis induced by recombinant human VEGF (rhVEGF; R\&D Systems, Minneapolis, 
MN, USA), tube formation, scratch wounding migration, and cell proliferation assays were performed. Apatinib stock solution ( $1 \mathrm{mM}$ in dimethyl sulfoxide [DMSO]) was diluted to the working concentration $(1 \mu \mathrm{M})$ in endothelial basal medium (EBM; Lonza). For the tube formation assay, HUVECs were seeded at 40,000 cells/well in 24-well plates coated with Matrigel (BD Bioscience, Bedford, MA, USA). Cells were then treated with EBM containing 1\% fetal bovine serum (FBS, Lonza), supplemented with rhVEGF (100 ng/mL), apatinib $(1 \mu \mathrm{M})$, Apa-HSA-PEG nanoparticles containing the equivalent amount of apatinib, or a combination of these treatments. After incubation for $6 \mathrm{~h}$, tube networks were imaged in four random microscopic fields and were quantified by measuring the tube lengths. For the scratch wounding migration assay, HUVECs were grown in a monolayer culture until $100 \%$ confluency. Endothelial monolayers were scratched using pipette tips and washed with PBS to remove dislodged cells and the medium. Cells were then incubated with EBM containing 1\% FBS, supplemented with rhVEGF (100 ng/mL), apatinib (1 $\mu \mathrm{M})$, Apa-HSA-PEG nanoparticles containing the equivalent amount of apatinib, or a combination of these treatments for $24 \mathrm{~h}$. Cell migration was monitored using optical microscopy and quantified by measuring the number of cells that had migrated from the wound edges. For the cell proliferation assay, HUVECs were seeded at 10,000 cells/well in gelatin-coated 96-well plates and were incubated with EBM containing 1\% FBS, supplemented with rhVEGF (100 ng/mL), apatinib $(1 \mu \mathrm{M})$, Apa-HSA-PEG nanoparticles containing the equivalent amount of apatinib, or a combination of these treatments for 2 days. Cell proliferation was assessed using the Cell Counting Kit-8 (CCK-8) assay (Dojindo Molecular Technology, Inc., Rockville, MD, USA). Briefly, $10 \mu \mathrm{L}$ of CCK-8 solution was added to each well, and the 96-well plate was incubated at $37^{\circ} \mathrm{C}$ for $4 \mathrm{~h}$. Then, the optical density of each well was read at a wavelength of $450 \mathrm{~nm}$ to determine the number of viable cells using a microplate reader (BioTek Instruments, Seoul, Korea).

\section{Animals}

Sprague Dawley (SD) rats with a mean weight of $250 \mathrm{~g}$ (5 weeks old; Nara Biotech, Seoul, Korea) were used in these studies. All animal studies were approved by the SKKU School of Pharmacy Institutional Animal Care and Use Committee in accordance with the ethical guidelines of the SKKU School of Pharmacy and the Association for Research in Vision and Ophthalmology statement regarding the use of animals in ophthalmic and vision research. The animals were maintained under a controlled 12-h light/dark cycle at an ambient temperature and had free access to water and standard rodent chow.

\section{Rat model of alkali burn injury-induced corneal neovascularization}

$\mathrm{SD}$ rats were anesthetized by an intraperitoneal injection of ketamine hydrochloride $(20 \mathrm{mg} / \mathrm{kg})$ and xylazine hydrochloride $(2.5 \mathrm{mg} / \mathrm{kg})$. The center of the right cornea was cauterized by pressing an applicator stick coated with $75 \%$ silver nitrate and $25 \%$ potassium nitrate for $20 \mathrm{~s}$ (day 0 ). Apatinib stock solution (5 $\mathrm{M}$ in DMSO) was diluted to $5 \mathrm{mM}$ with PBS for injections. Each injured rat eye received a subconjunctival injection of $50 \mu \mathrm{L}$ of solution of apatinib (5 mM, $124 \mu \mathrm{g} /$ eye), Apa-HSA-PEG nanoparticles containing an equivalent amount of apatinib, or PBS on day 3 and 5.

Seven days after the final injection, the eyes were examined and imaged using a stereoscopic microscope (Olympus, Tokyo, Japan). The images were used to measure the corneal surface covered with blood vessels. The area of corneal neovascularization was calculated as a percentage of the total corneal area using ImageJ software (version 1.42, National Institute of Health, MD, USA). For additional analysis of corneal neovascularization, anesthetized rats were perfused with $50 \mathrm{mg} / \mathrm{mL}$ fluorescein isothiocyanate (FITC)-labeled dextran (MW 40,000; Sigma). ${ }^{19}$ The animals were then euthanized by cervical dislocation. The eyes were enucleated and washed twice with PBS. After the posterior segment of the eyeball was removed, the anterior segment was mounted on a glass slide and observed using a fluorescence microscope (IX81-ZDC, Olympus). Corneal neovascularization was quantitatively evaluated by measuring the net fluorescence intensity of each image above the fluorescence intensity value of the background area free from blood vessels, using the ImageJ software.

\section{Intracorneal distribution of Apa-HSA- PEG nanoparticles}

The intracorneal distribution of HSA-PEG nanoparticles after a subconjunctival injection was observed using a fluorescent probe, 1,1'-dioctadecyl-3,3,3',3'-tetramethylindodicarbocya nine perchlorate (DiD) as a model hydrophobic drug. DiD and DiD-loaded HSA-PEG (DiD-HSA-PEG) nanoparticles were subconjunctivally injected into the eyes of alkali burninjured rats. After the desired treatment period (3, 6, and $24 \mathrm{~h}$ after the injection), the animals were euthanized by cervical dislocation, and their eyes were enucleated, fixed, 
and embedded in optimal cutting temperature compound (Sakura Finetek, Tokyo, Japan). Cryosections (10 $\mu \mathrm{m}$ thick) were cut, washed twice with deionized water, and stained with 4',6-diamidino-2-phenylindole solution for $10 \mathrm{~min}$ at room temperature. The intracorneal distribution of DiD and DiD-HSA-PEG nanoparticles was observed using a confocal microscope (Leica TCS SP5, Wetzlar, Germany).

\section{Statistical analysis}

All data are presented as mean \pm standard error of the mean. A one-way analysis of variance followed by Bonferroni's post hoc multiple comparison test was used to determine the differences between multiple groups. A $P$-value $<0.05$ was considered statistically significant, and the number of samples in each experiment is indicated by $n$.

\section{Results and discussion}

\section{Apa-HSA-PEG nanoparticles block VEGF-mediated angiogenesis in human endothelial cells}

Apatinib is a novel small-molecule inhibitor targeting the intracellular ATP-binding site of VEGFR2 tyrosine kinase, and it is currently under investigation in clinical trials as a third-line treatment option for advanced metastatic gastric cancer. In our previous study, we successfully formulated apatinib, a poorly water-soluble drug, as self-assembled nanoparticle in an aqueous medium with an HSA-PEG conjugate by a solvent-free film casting and rehydration method. ${ }^{18}$ Hydrophobic apatinib is expected to be localized at the hydrophobic domains of HSA-PEG that are likely exposed during the solvent evaporation process at an elevated temperature. The entrapment might effectively prevent the formation and growth of drug crystals in the film. The surface of the nanoparticles would be stabilized by the PEG segments that form the outermost hydrophilic layer. The Apa-HSAPEG nanoparticles showed spherical morphology, and their hydrodynamic diameters before and after lyophilization were 131.7 \pm 3.5 and 169.6 $\pm 5.2 \mathrm{~nm}$, respectively (Figure S1). The drug loading amount and encapsulation efficiency of Apa-HSA-PEG nanoparticle were $5.4(\mathrm{w} / \mathrm{w})$ and $77.1 \%$, respectively. In vitro drug release experiment revealed that apatinib was released from Apa-HSA-PEG nanoparticles in a sustained manner (Figure S2).

To analyze the antiangiogenic effects of Apa-HSA-PEG nanoparticles, we first evaluated their inhibitory effect on VEGF-induced angiogenic responses including Matrigel tube formation, scratch wounding migration, and cell proliferation. Figure 1 shows that VEGF significantly enhanced the formation of tubular network in HUVECs, while co-treatment with Apa-HSA-PEG nanoparticles (equivalent to $1 \mu \mathrm{M}$ apatinib) completely inhibited this effect. The inhibitory effect of Apa-HSA-PEG nanoparticles was similar to that of free apatinib at the same concentration. The scratch wounding migration assay also demonstrated that Apa-HSA-PEG nanoparticles and free apatinib exhibited comparable activity in blocking VEGF-mediated mobility of endothelial cells (Figure 2). In the cell proliferation experiment using the CCK-8 assay, VEGF also substantially enhanced endothelial cell proliferation (Figure 3 ). The VEGF-induced increase in cell proliferation was almost completely blocked by cotreatment with Apa-HSA-PEG nanoparticles or free apatinib. These results indicate that Apa-HSA-PEG nanoparticles
A

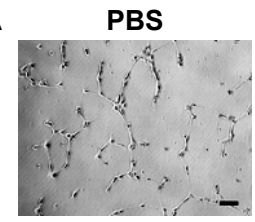

VEGF +

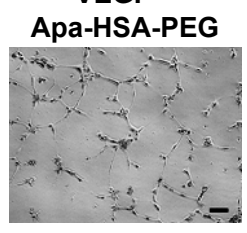

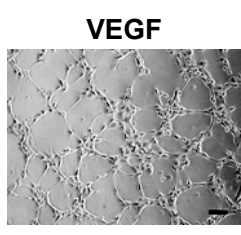

VEGF + Apa

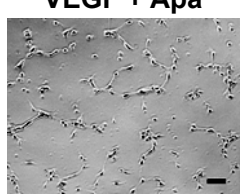

B

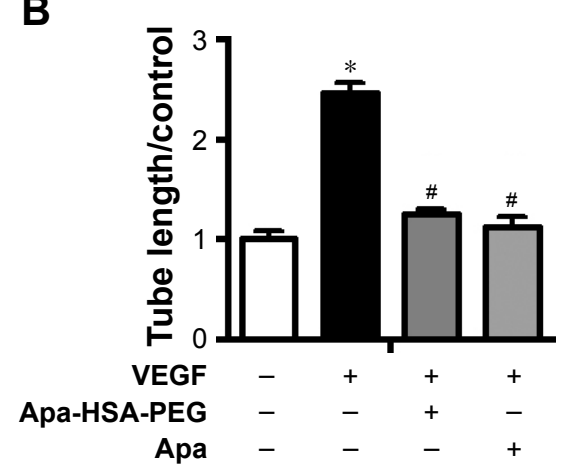

Figure I Apa-HSA-PEG nanoparticles suppress VEGF-induced tube formation in human endothelial cells.

Notes: (A) Representative images of tube formation and (B) quantification of tube length. HUVECs were seeded onto Matrigel and treated with PBS, Apa (I $\mu$ M), or ApaHSA-PEG nanoparticles containing an equivalent amount of Apa in the presence or absence of rhVEGF ( $100 \mathrm{ng} / \mathrm{mL})$ for $6 \mathrm{~h}$. Tube networks were quantified by measuring tube length in four random microscopic fields. Data were normalized relative to values of the corresponding controls and are expressed as fold change \pm SEM relative to PBS control $(* P<0.05$ vs PBS control and $\# P<0.05$ vs VEGF only, $n=5)$. Scale bars $=50 \mu \mathrm{m}$.

Abbreviations: Apa-HSA-PEG, apatinib-loaded human serum albumin-conjugated polyethylene glycol; VEGF, vascular endothelial growth factor; HUVECs, human umbilical vein endothelial cells; PBS, phosphate-buffered saline; Apa, apatinib; rhVEGF, recombinant human VEGF; SEM, standard error of the mean. 


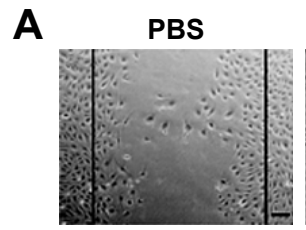

VEGF +

Apa-HSA-PEG

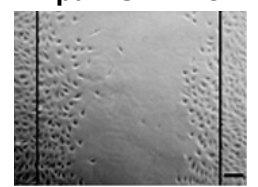

VEGF

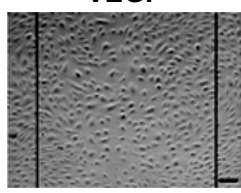

VEGF + Apa

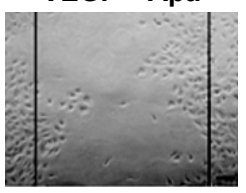

Figure 2 Apa-HSA-PEG nanoparticles inhibit VEGF-induced wounding migration of human endothelial cells.

Notes: (A) Representative images of scratch wounding migration and (B) quantification of migrating cell number. Confluent HUVEC monolayers grown in six-well plates were scratched and treated with PBS, Apa (I $\mu \mathrm{M})$, or Apa-HSA-PEG nanoparticles containing an equivalent amount of Apa in the presence or absence of rhVEGF (I00 ng/mL) for $24 \mathrm{~h}$. Scratch wounding migration was quantified by determining the number of cells that migrated from the wound edges (black lines). Data are expressed as mean \pm SEM ( $* P<0.05$ vs PBS control and ${ }^{\#} P<0.05$ vs VEGF only, $\left.n=5\right)$. Scale bars $=50 \mu \mathrm{m}$.

Abbreviations: Apa-HSA-PEG, apatinib-loaded human serum albumin-conjugated polyethylene glycol; VEGF, vascular endothelial growth factor; HUVEC, human umbilical vein endothelial cell; PBS, phosphate-buffered saline; Apa, apatinib; rhVEGF, recombinant human VEGF; SEM, standard error of the mean.

substantially inhibit VEGF-induced angiogenesis in HUVECs with efficiency similar to that of free apatinib.

\section{Apa-HSA-PEG nanoparticles inhibits corneal neovascularization in rats}

The cornea is normally avascular and receives oxygen and nutrients via diffusion from the tear fluid through the aqueous humor. However, infectious diseases, injury, and inflammation promote the ingrowth of blood vessels from the limbal vascular plexus into the center of the cornea, which leads to an increase in corneal opacity and affects visual acuity. Because VEGF has been considered a key trigger of

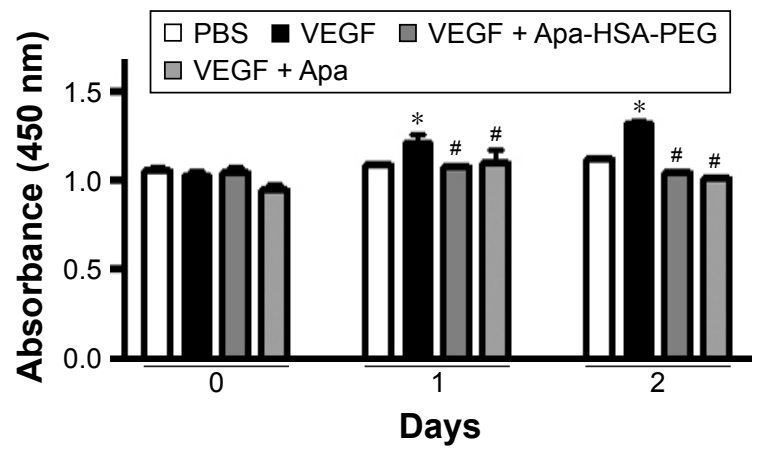

Figure 3 Apa-HSA-PEG nanoparticles block VEGF-induced proliferation of human endothelial cells.

Notes: HUVECs were treated with PBS, Apa (I $\mu \mathrm{M})$, or Apa-HSA-PEG nanoparticles containing an equivalent amount of Apa in the presence or absence of rhVEGF $(100 \mathrm{ng} / \mathrm{mL})$ for I or 2 days. Cell proliferation was analyzed using the CCK-8 assay. Absorbance values are expressed as mean \pm SEM ( ${ }^{* P}<0.05$ vs PBS, ${ }^{*} P<0.05$ vs VEGF only, $n=6$ ).

Abbreviations: Apa-HSA-PEG, apatinib-loaded human serum albumin-conjugated polyethylene glycol; VEGF, vascular endothelial growth factor; HUVEC, human umbilical vein endothelial cell; PBS, phosphate-buffered saline; Apa, apatinib; rhVEGF, recombinant human VEGF; CCK-8, Cell Counting Kit-8; SEM, standard error of the mean. neovascularization of the cornea, we determined whether Apa-HSA-PEG nanoparticles could inhibit corneal neovascularization in an animal model of alkali burn injury. On day 3 and 5 after silver nitrate cautery, rats received subconjunctival injections of PBS, free apatinib solution, or Apa-HSA-PEG nanoparticles containing an equivalent amount of apatinib. Seven days after the final injection, the corneas treated with PBS exhibited excessive ingrowth of neovessels from the limbus into the central corneal stroma, and those treated with free apatinib did not show significant inhibition of neovascularization compared with those in the PBS-treated control (Figure 4A and B). By contrast, a subconjunctival injection of Apa-HSA-PEG nanoparticles substantially decreased new blood vessel formation in the cornea. For an additional quantitative analysis of anti-angiogenic effects, the extent of corneal neovascularization was assessed by perfusing rats with FITC-labeled dextrans and measuring their fluorescence intensity in the cornea. The analysis showed a significant reduction in the relative fluorescence intensity of Apa-HSAPEG nanoparticle-injected corneas compared with those of the free apatinib- or PBS-injected controls (Figure 4C and D). These data suggest that Apa-HSA-PEG nanoparticles exert a substantially greater inhibitory effect on in vivo corneal neovascularization than the free apatinib solution does.

\section{In vivo corneal distribution of HSA-PEG nanoparticles}

In the alkali burn injury animal model, new blood vessels are prominently formed in the anterior part of the corneal stroma, and so, subconjunctivally injected molecules should migrate and distribute in the corneal stroma to inhibit 
A
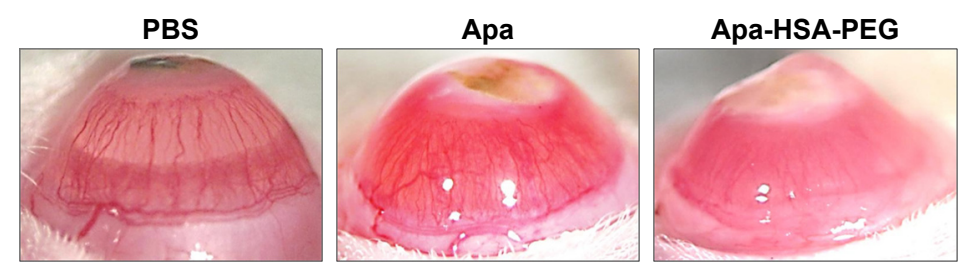

C
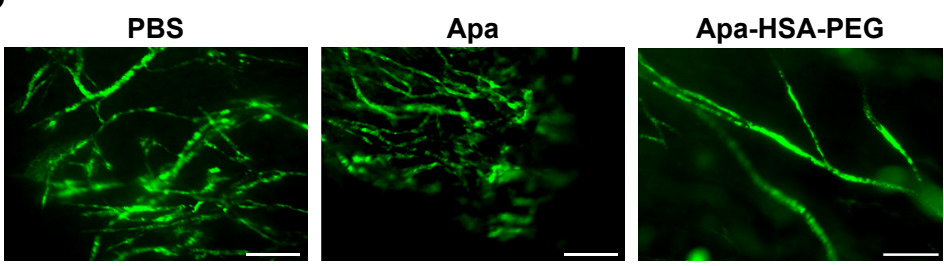

B

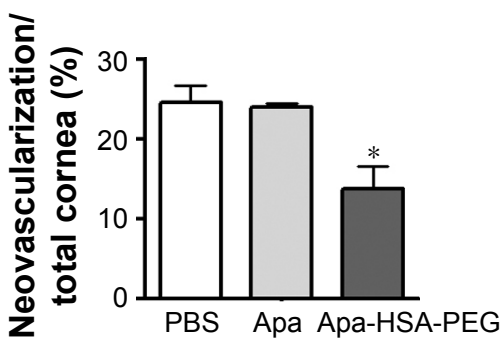

D

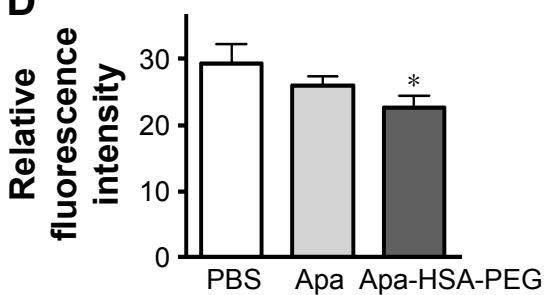

Figure 4 Apa-HSA-PEG nanoparticles inhibit alkali burn-induced corneal neovascularization in rats.

Notes: The centers of the right corneas of SD rats were cauterized using applicator sticks composed of silver nitrate and potassium nitrate. On day 3 and 5 after cauterization, rats received subconjunctival injections of PBS, Apa (I24 $\mathrm{gg} / \mathrm{eye}$ ), or Apa-HSA-PEG nanoparticles containing an equivalent amount of Apa. Corneal neovascularization was assessed 7 days after injection. (A) Representative stereoscopic images of corneas treated with PBS, Apa, and Apa-HSA-PEG nanoparticles are shown. Original magnification $8 \times$. (B) Area of neovascularization was measured in pixels using Imagel software and calculated as a percentage of the total corneal area. Data are expressed as the mean \pm SEM ( $* P<0.05$ vs PBS control, $n=3$ mice/group). (C) Representative fluorescence microscopic images of corneal vasculature and (D) quantification of fluorescence intensities of cornea sections. Corneal neovascularization was visualized by perfusion with FITC-labeled dextrans. Relative fluorescence intensity of FITC-labeled dextrans in the corneas was assessed using Imagej software. Data are expressed as mean \pm SEM $(* P<0.05$ vs PBS control, $n=4$ mice/group). Scale bars $=200 \mu m$.

Abbreviations: Apa-HSA-PEG, apatinib-loaded human serum albumin-conjugated polyethylene glycol; SD, Sprague Dawley; PBS, phosphate-buffered saline; Apa, apatinib; SEM, standard error of the mean; FITC, fluorescein isothiocyanate.

neovascularization. Moreover, the stroma, which comprises $90 \%$ of the corneal thickness, is highly hydrated and acts as a significant barrier against the permeation of hydrophobic molecules. Therefore, we hypothesized that the encapsulation of water-insoluble apatinib in HSA-PEG nanoparticle might improve the distribution of apatinib in the hydrophilic stromal layer, thereby increasing the efficacy of apatinib to prevent corneal neovascularization compared with that of free drug solution. For the analysis of the in vivo corneal distribution of HSA-PEG nanoparticles, an alkylated fluorescent dye, DiD, was used as a water-insoluble model drug. When the red fluorescent DiD-loaded HSA-PEG nanoparticles and free $\mathrm{DiD}$ were separately injected into the subconjunctiva of alkali burn-injured rats, a substantial level of red fluorescence was detected in the corneal stroma of rats treated with nanoparticles after $24 \mathrm{~h}$, whereas little fluorescence was observed in the corneal stroma of rats injected with free DiD (Figure 5). This result suggests that HSA-PEG nanoparticle may facilitate the distribution of hydrophobic apatinib in the corneal stromal layer where neovascularization occurs.

\section{Conclusion}

The cornea maintains a balance between angiogenic and antiangiogenic factors, which allows it to maintain its avascularity that is necessary for the preservation of optimal vision. In particular, the corneal stroma, which accounts for $90 \%$ of the corneal thickness, should remain avascular for corneal transparency. However, the cornea is vascularized in several pathological conditions where this balance is disrupted toward angiogenesis, impairing vision through the loss of corneal clarity. A recent therapeutic strategy for corneal neovascularization involves blocking the angiogenic action of VEGF using small-molecule inhibitors of VEGFR2 whose kinase activity mediates critical angiogenic intracellular signaling. For example, treatment with SU5416, a selective inhibitor of VEGFR2 tyrosine kinase, had an inhibitory effect on alkali burn-induced corneal neovascularization in mice. ${ }^{20}$ Regorafenib, an inhibitor of multiple tyrosine kinases including VEGFRs, inhibited corneal neovascularization in a similar animal model. ${ }^{21}$ Treatment with oral small-molecule inhibitors of VEGFR reduced suture-induced hemangiogenesis in the cornea and enhanced the survival of allogenic corneal transplants in rats. ${ }^{17}$ However, many VEGFR2 tyrosine kinase inhibitors including the abovementioned agents have low water solubility, which is a major obstacle for their ocular delivery, distribution, and therapeutic action in the hydrophilic corneal stroma where neovascularization occurs. Therefore, drug carriers are required for effective ocular delivery of poorly water-soluble inhibitors. Nanotechnology may offer a solution by providing an amendable 


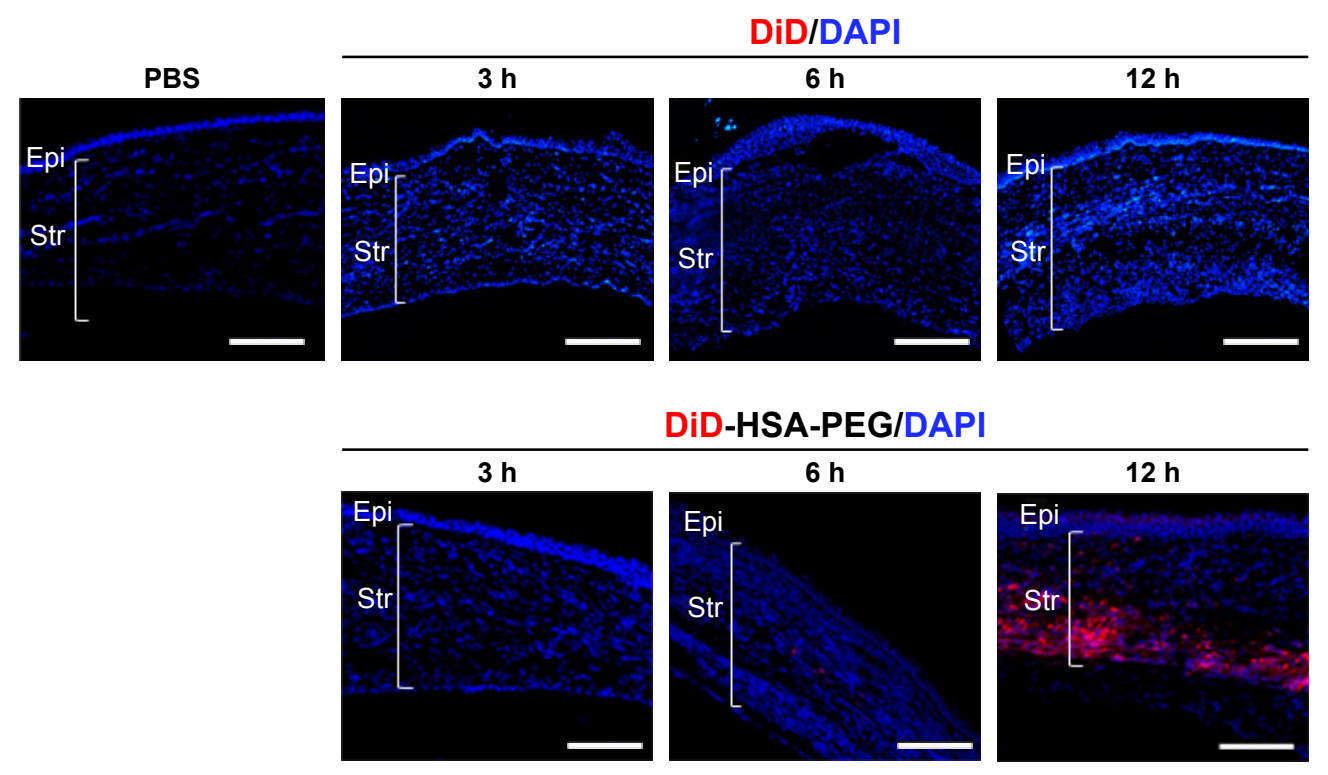

Figure 5 Intracorneal distribution of DiD-HSA-PEG nanoparticles and DiD after subconjunctival injection into alkali burn-injured rats.

Notes: Representative fluorescence microscopic images show central regions of corneas harvested 3, 6, or 24 h after subconjunctival injection of DiD-HSA-PEG nanoparticles, DiD, or PBS into alkali burn-injured rats. Fluorescent DiD (red) was used a model hydrophobic drug similar to apatinib. Cell nuclei were stained with DAPI (blue). Scale bars $=100 \mu \mathrm{m}$.

Abbreviations: DiD, I, I'-dioctadecyl-3,3,3',3'-tetramethylindodicarbocyanine perchlorate; DiD-HSA-PEG, DiD-loaded human serum albumin-conjugated polyethylene glycol; DAPI, 4',6-diamidino-2-phenylindole; PBS, phosphate-buffered saline; Epi, corneal epithelium; Str, stroma.

strategy for improving drug solubilization and delivery to the stroma, thereby enhancing the efficacy of corneal neovascularization treatment.

In the present study, we demonstrated the therapeutic potential of apatinib in the treatment of pathological ocular neovascularization. Apatinib is water insoluble, similar to many small-molecule tyrosine kinase inhibitors. Therefore, HSA-PEG nanoparticles were used to encapsulate apatinib and improve its ocular delivery and efficacy, which is schematically illustrated in Figure 6. Among the numerous types of nanoparticles, HSA-PEG is an excellent drug delivery carrier. HSA is a natural protein carrier in the human blood and,

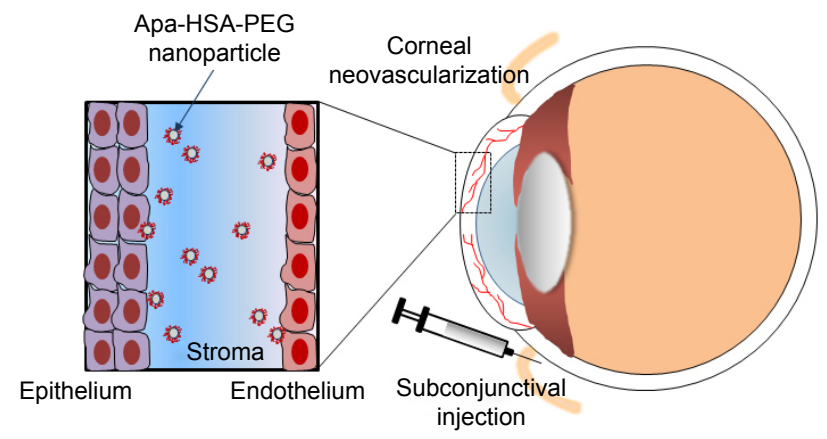

Figure 6 Schematic illustration of intraocular delivery of Apa-HSA-PEG nanoparticles in an animal model of corneal neovascularization.

Note: A subconjunctival injection of Apa-HSA-PEG nanoparticles and their biodistribution in the corneal stroma of rats with corneal neovascularization.

Abbreviation: Apa-HSA-PEG, apatinib-loaded human serum albumin-conjugated polyethylene glycol. therefore, is a safe biomaterial for delivering water-insoluble drugs. ${ }^{22,23}$ For example, HSA-based nanoparticles of waterinsoluble paclitaxel were approved by the US Food and Drug Administration for the treatment of breast and pancreatic cancers. ${ }^{24}$ In the present study, HSA was modified with PEG to enhance the solubility and biocompatibility of the nanoparticles, which may have improved the biodistribution of water-insoluble apatinib in the hydrophilic corneal stroma layer. In this study, we compared the inhibitory effects of Apa-HSA-PEG nanoparticles and free apatinib on VEGF-induced in vitro angiogenesis, and on alkali burn injury-induced corneal neovascularization in rats. In the in vitro experiments, Apa-HSA-PEG nanoparticles and free apatinib exhibited similar efficiency in blocking VEGFinduced endothelial cell proliferation, migration, and tube formation. By contrast, the in vivo study showed that the subconjunctival injection of Apa-HSA-PEG nanoparticles significantly reduced corneal neovascularization, while the injection of free apatinib solution did not show a remarkable decrease in neovascularization. This improved in vivo efficacy of Apa-HSA-PEG nanoparticles over that of free apatinib might be explained by the biodistribution data, which showed that a substantial number of HSA-PEG nanoparticles reached the corneal stroma within $24 \mathrm{~h}$ after injection. These results demonstrate the advantage of using Apa-HSA-PEG nanoparticles over free apatinib for the prevention of corneal neovascularization. In addition, small-molecule drugs are 
rapidly eliminated from ocular tissues following a subconjunctival injection. As this rapid clearance limits the duration of therapeutic drug concentration in ocular tissues and necessitates frequent drug injection, a sustained drug delivery is expected to enhance the ocular bioavailability of drugs and reduce the frequency of drug administration, which is important to improve patient compliance. For this reason, HSA-PEG nanoparticles can provide a sustained release of drug to ocular tissues at effective levels, which can increase therapeutic outcome and relieve poor patient compliance. We conclude that the anticancer drug apatinib may be a potential therapy for corneal neovascularization using HSA-PEG nanoparticles as a drug delivery carrier.

\section{Acknowledgment}

This research was supported by the Bio \& Medical Technology Development Program of National Research Foundation of Korea grants funded by the Korea government (2012M3A9C6050368, 2015R1D1A1A02061724, and 2016R1A2B4015056).

\section{Disclosure}

The authors report no conflicts of interest in this work.

\section{References}

1. Azar DT. Corneal angiogenic privilege: angiogenic and antiangiogenic factors in corneal avascularity, vasculogenesis, and wound healing (an American Ophthalmological Society thesis). Trans Am Ophthalmol Soc. 2006;104:264-302.

2. Ellenberg D, Azar DT, Hallak JA, et al. Novel aspects of corneal angiogenic and lymphangiogenic privilege. Prog Retin Eye Res. 2010; 29(3):208-248.

3. Ambati BK, Nozaki M, Singh N, et al. Corneal avascularity is due to soluble VEGF receptor-1. Nature. 2006;443(7114):993-997.

4. Ambati BK, Patterson E, Jani $P$, et al. Soluble vascular endothelial growth factor receptor-1 contributes to the corneal antiangiogenic barrier. $\mathrm{BrJ}$ Ophthalmol. 2007;91(4):505-508.

5. Amano S, Rohan R, Kuroki M, Tolentino M, Adamis AP. Requirement for vascular endothelial growth factor in wound- and inflammationrelated corneal neovascularization. Invest Ophthalmol Vis Sci. 1998; 39(1):18-22.

6. Kvanta A, Sarman S, Fagerholm P, Seregard S, Steen B. Expression of matrix metalloproteinase-2 (MMP-2) and vascular endothelial growth factor (VEGF) in inflammation-associated corneal neovascularization. Exp Eye Res. 2000;70(4):419-428.
7. Mastyugin V, Mosaed S, Bonazzi A, Dunn MW, Schwartzman ML. Corneal epithelial VEGF and cytochrome P450 4B1 expression in a rabbit model of closed eye contact lens wear. Curr Eye Res. 2001;23(1): $1-10$.

8. Bahar I, Kaiserman I, McAllum P, Rootman D, Slomovic A. Subconjunctival bevacizumab injection for corneal neovascularization in recurrent pterygium. Curr Eye Res. 2008;33(1):23-28.

9. Carrasco MA. Subconjunctival bevacizumab for corneal neovascularization in herpetic stromal keratitis. Cornea. 2008;27(6):743-745.

10. Doctor PP, Bhat PV, Foster CS. Subconjunctival bevacizumab for corneal neovascularization. Cornea. 2008;27(9):992-995.

11. Gerten G. Bevacizumab (avastin) and argon laser to treat neovascularization in corneal transplant surgery. Cornea. 2008;27(10):1195-1199.

12. Qian CX, Bahar I, Levinger E, Rootman D. Combined use of superficial keratectomy and subconjunctival bevacizumab injection for corneal neovascularization. Cornea. 2008;27(9):1090-1092.

13. Uy HS, Chan PS, Ang RE. Topical bevacizumab and ocular surface neovascularization in patients with Stevens-Johnson syndrome. Cornea. 2008;27(1):70-73.

14. Wu PC, Kuo HK, Tai MH, Shin SJ. Topical bevacizumab eyedrops for limbal-conjunctival neovascularization in impending recurrent pterygium. Cornea. 2009;28(1):103-104.

15. Olsson AK, Dimberg A, Kreuger J, Claesson-Welsh L. VEGF receptor signalling - in control of vascular function. Nat Rev Mol Cell Biol. 2006;7(5):359-371.

16. Chang JH, Garg NK, Lunde E, Han KY, Jain S, Azar DT. Corneal neovascularization: an anti-VEGF therapy review. Surv Ophthalmol. 2012;57(5):415-429.

17. Hos D, Bock F, Dietrich T, et al. Inflammatory corneal (lymph)angiogenesis is blocked by VEGFR-tyrosine kinase inhibitor ZK 261991, resulting in improved graft survival after corneal transplantation. Invest Ophthalmol Vis Sci. 2008;49(5):1836-1842.

18. Jeong JH, Nguyen HK, Lee JE, Suh W. Therapeutic effect of apatinibloaded nanoparticles on diabetes-induced retinal vascular leakage. Int J Nanomedicine. 2016;11:3101-3109.

19. Kim SR, Suh W. Beneficial effects of the Src inhibitor, dasatinib, on breakdown of the blood-retinal barrier. Arch Pharm Res. 2017;40(2): 197-203.

20. Keskin U, Totan Y, Karadağ R, Erdurmuş M, Aydin B. Inhibitory effects of SU5416, a selective vascular endothelial growth factor receptor tyrosine kinase inhibitor, on experimental corneal neovascularization. Ophthalmic Res. 2012;47(1):13-18.

21. Onder HI, Erdurmus M, Bucak YY, Simavli H, Oktay M, Kukner AS. Inhibitory effects of regorafenib, a multiple tyrosine kinase inhibitor, on corneal neovascularization. Int J Ophthalmol. 2014;7(2):220-225.

22. Kompella UB, Amrite AC, Pacha Ravi R, Durazo SA. Nanomedicines for back of the eye drug delivery, gene delivery, and imaging. Prog Retin Eye Res. 2013;36:172-198.

23. Kragh-Hansen U, Chuang VT, Otagiri M. Practical aspects of the ligand-binding and enzymatic properties of human serum albumin. Biol Pharm Bull. 2002;25(6):695-704.

24. Sparreboom A, Scripture CD, Trieu V, et al. Comparative preclinical and clinical pharmacokinetics of a cremophor-free, nanoparticle albuminbound paclitaxel (ABI-007) and paclitaxel formulated in Cremophor (Taxol). Clin Cancer Res. 2005;11(11):4136-4143. 


\section{Supplementary materials}
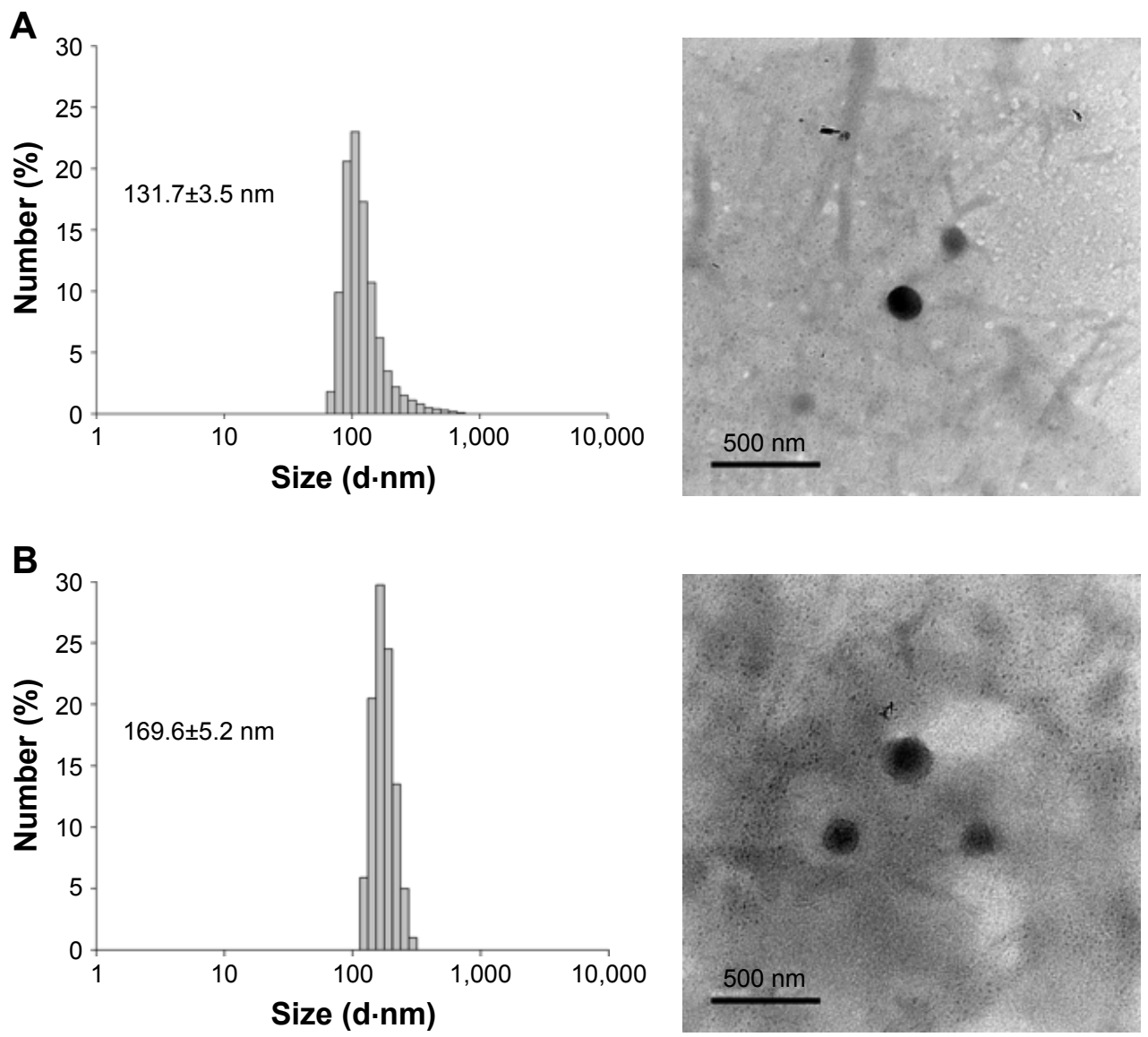

Figure SI Size distribution and morphology of Apa-HSA-PEG nanoparticles before (A) and after (B) lyophilization. Abbreviation: Apa-HSA-PEG, apatinib-loaded human serum albumin-conjugated polyethylene glycol.

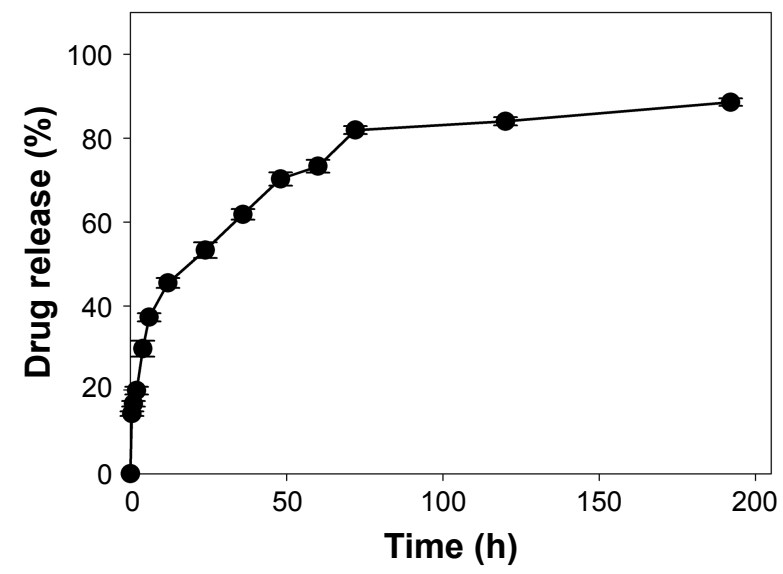

Figure S2 Cumulative release of apatinib from Apa-HSA-PEG in an aqueous solution.

Abbreviation: Apa-HSA-PEG, apatinib-loaded human serum albumin-conjugated polyethylene glycol. 


\section{Publish your work in this journal}

The International Journal of Nanomedicine is an international, peerreviewed journal focusing on the application of nanotechnology in diagnostics, therapeutics, and drug delivery systems throughout the biomedical field. This journal is indexed on PubMed Central,

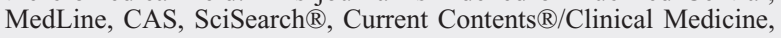

Journal Citation Reports/Science Edition, EMBase, Scopus and the Elsevier Bibliographic databases. The manuscript management system is completely online and includes a very quick and fair peer-review system, which is all easy to use. Visit http://www.dovepress.com/ testimonials.php to read real quotes from published authors.

Submit your manuscript here: http://www.dovepress.com/international-journal-of-nanomedicine-journal 